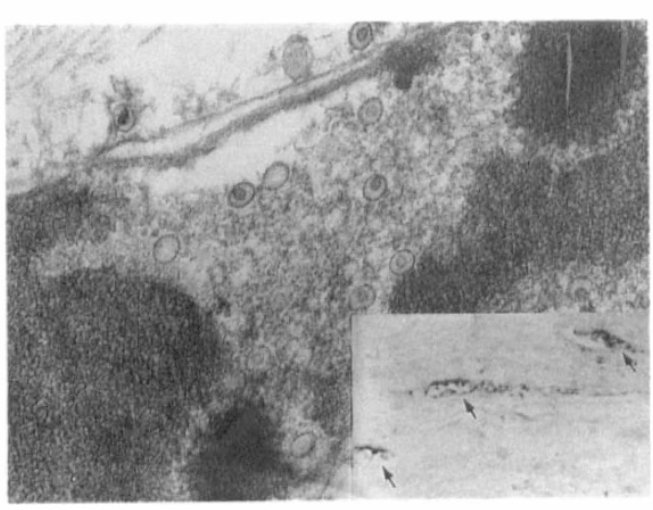

Fig. 1. Electron micrograph showing herpes simplex viral particles within the nucleus and adjacent to cytoplasm of a keratocyte $(\times 54,000)$. Inset. HSV 1 nucleic acid revealed by in situ hybridisation $(\times 200)$, arrows. The peripheral localisation of the probe on the inner aspect of nuclear membrane is consistent with active transcription.

ocular pathogen, we are unaware of any firm clinical evidence to suggest that herpes simplex virus (HSV) has been transmitted in this way. We report the identification of this agent in the keratocytes of a corneal donor.

The donor was a single, fit 42 year old male who was ventilated for eight days after a head injury resulting from a road traffic accident. The eyes were macroscopically normal and showed no obvious abnormality on slit-lamp examination after removal and before placement of the corneo-scleral discs in organ culture medium. Routine bacteriology at eight days in culture failed to reveal any organisms. However, assessment prior to transplantation revealed that both endothelial layers were necrotic or absent. A sample of the storage medium from the left cornea was cultured for virus and both corneas were fixed for microscopic examination. Culture in three separate cell lines revealed typical cytopathic effect typical of herpes simplex type I. Light microscopy of both corneas revealed intranuclear inclusions in a large number of keratocytes. Electron microscopy revealed large numbers of herpes virus particles in and around keratocytes (Fig. 1). In situ DNA hybridisation using a probe to herpes simplex type I thymidine kinase revealed widespread localisation of the probe in the nuclei of many stromal cells (Fig. 1, inset). The donor had complement fixation titres against HSV of less than 1/10, but was weakly positive by radioimmunosorbent assay. This report has highlighted the usefulness of organ culture in screening donor tissue for infection. The observation of unexpected endothelial cell loss has been made by several workers in different organ culture eye banks (personal communications) but hitherto HSV type I has not been incriminated. We are currently seeking further evidence to demonstrate that this pathogen is transmissible by corneal transplantation.
A. B. Tullo
B. Marcyniuk
R. Bonshek
C. Dennett
G. M. Cleator
A. G. Lewis
P. E. Klapper
Manchester Royal Eye Hospital,
Oxford Road,
Manchester.

References

${ }^{1}$ O'Day DM. Diseases potentially transmitted through corneal transplantation. Ophthalmology 1989, 96: 1133-8.

${ }^{2}$ Gandhi SS, Lamberts DW, Perry HD: Donor to Host Transmission of disease via Corneal Transplantation. Surv Ophthalmol 1981, 25: 306-11.

${ }^{3}$ Gastaud P, Baudouin CH, Ousan D: Detection of HBs antigen, DNA polymerase activity and hepatitis B virus. DNA in tears: reference to hepatitis B transmission by tears. Br J Ophthalmol 1989, 73: $333-6$.

${ }^{4}$ Salahuddin SZ, Palestine AG, Heck E, Ablashi D, Luckenbach M, McCulley JP, Nussenblatt RB: Isolation of human T-cell leukemia/lymphotropic virus type III from the cornea. Am J Ophthalmol 1986, 101-49.

Sir,

\section{Extensive chorioretinopathy associated with very low dose Thioridazine}

A case is reported in which a 57 year old woman presented with an extensive form of chorioretinal dystrophy following a short course of low dose thioridazine.

Pigmentary retinopathy is a well recognized side effect of high daily dose thioridazine treatment (above $800 \mathrm{mg} / \mathrm{day})^{1}$ and less frequently with lower doses. ${ }^{1,2}$ Most cases reported with low daily dose toxicity present with rather mild pigmentary disturbances and some of these patients seem to have been taking the drug for relatively long periods. 


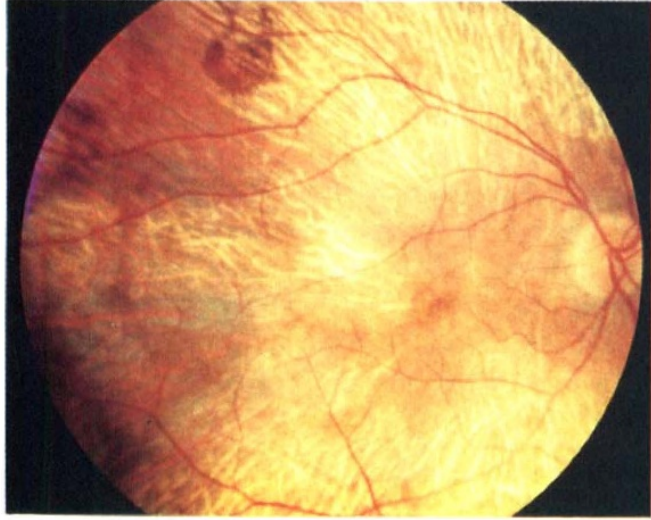

Fig. 1.

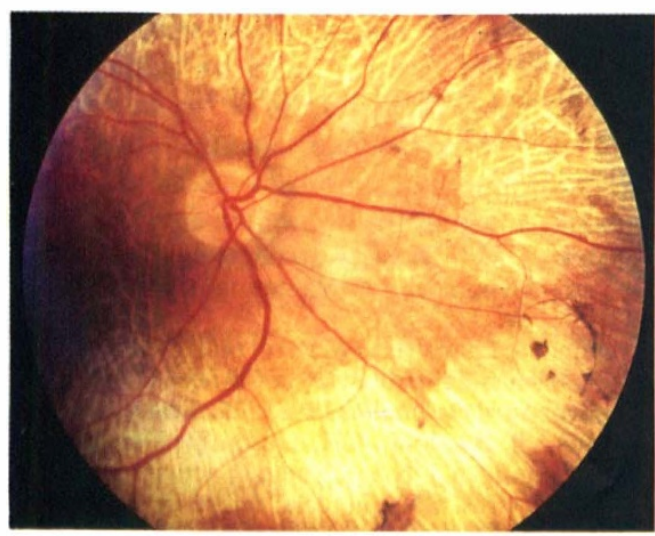

Fig. 3.

We believe this is the first reported case in which a total dose of $45 \mathrm{~g}$ given as a daily dose of $100 \mathrm{mg}$ with a 2-month increase to $400 \mathrm{mg}$ over a period of 270 days, has produced such extensive chorioretinal damage.

\section{Case Report}

A 57 year old woman presented to the clinic complaining of deterioration of visual acuity and night blindness over four months. There were no other symptoms and the patient did not recall any previous eye problems, confirmed by optician's reports and fundus examination by psychiatrists before the course of thioridazine treatment. Family history, confirmed by her only son, failed to reveal any known eye disorder for three generations. She had been treated for schizophrenia in the 1970s with a pre-frontal leucotomy, followed by several applications of electroconvulsive

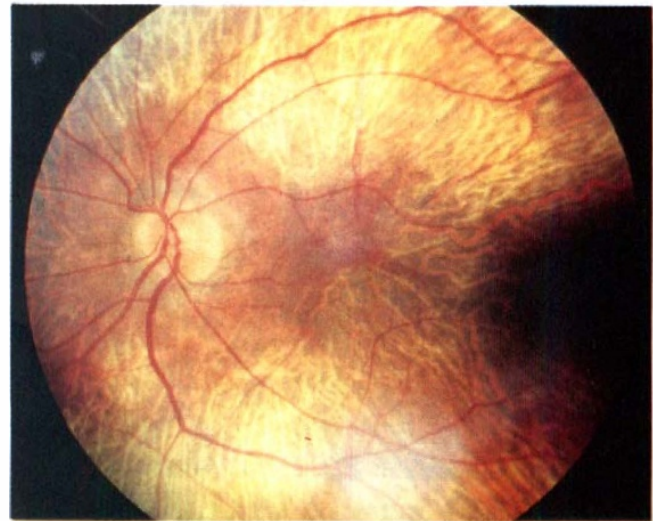

Fig. 2.

Figs. 1-3. Colour pictures showing extensive chorioretinal dystrophy in both eyes.

therapy. Since then she has been treated for depression with a combination maintenance course of chlorpromazine $75-100 \mathrm{mg}$ nocte, phenelzine $45-60 \mathrm{mg}$ daily and clomipramine hydrochloride $10-60 \mathrm{mg}$ daily on and off. Lithium carbonate at a maximum daily dose up to $750 \mathrm{mg}$ at times was added in 1983 . She has also had a course of thioridazine as described above which was stopped 12 months before the patient reported to us, when the psychiatrist noticed subtle pigmentary changes in the retinas of both eyes. She looked otherwise well, active and reportedly involved in social work. Findings included uncorrected visual acuity of $6 / 6$ in the right eye and 6/18 in the left eye (not improved with pin-hole or refraction), gross constriction of visual fields, normal anterior segments with clear lenses, normal appearance of the optic discs and retinal vasculature and extensive 


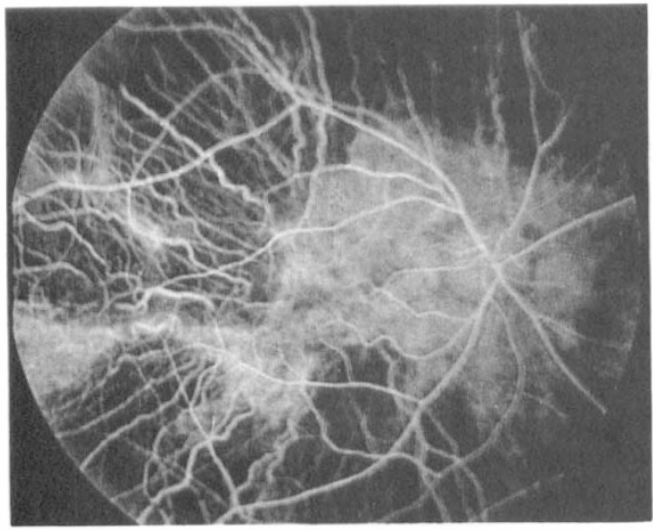

Fig. 4.

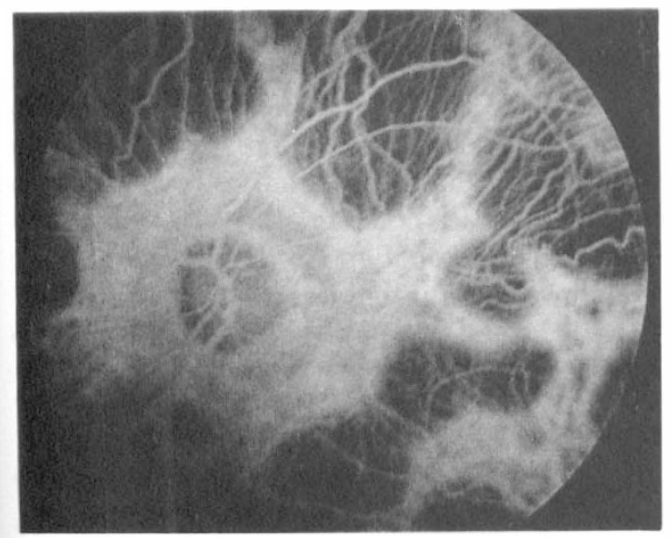

Fig. 6.

chorioretinal dystrophy with pigment clumping and very moderate sparing of the peripapillary and foveal area (Figs. 1-3). Fluorescein angiography revealed extensive areas of retinal pigment epithelium and choriocapillaris atrophy with reasonable choriocapillaris circulation in the peripapillary area seen through attenuated retinal pigment epithelium. ${ }^{3}$ Arterial filling seemed normal without any leakage (Figs. 3-6). ERG and EOG were very attenuated bilaterally, consistent with an extensive lesion of both retinas with involvement of outer retina and pigment epithelium (results included absent a and $b$ waves in both eyes to blue and white stimulus and reduced responses to a red photopic stimulus and to flicker on the flash ERG. Pattern ERG was also very attenuated. Arden index for the EOG revealed a percentage of $122 \%$ and $125 \%$ respectively to the right and left eyes).

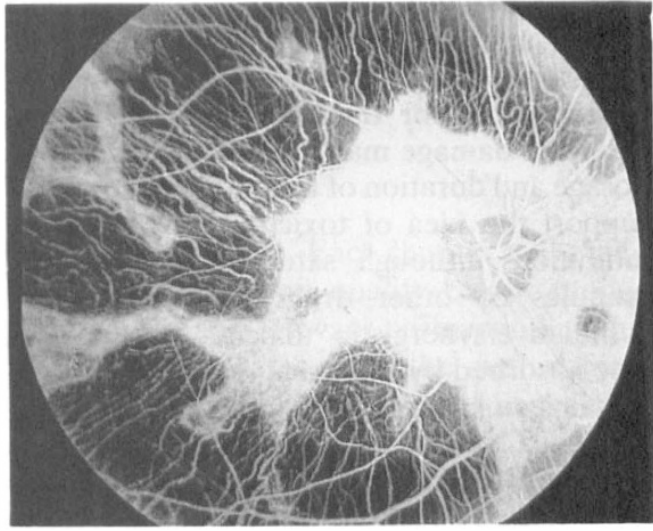

Fig. 5.

Figs. 4-6. Fundus fluorescein angiography: positive prints revealing extensive areas of retinal pigment epithelium and choriocapillaris atrophy with reasonable. choriocapillaris circulation in the peripapillary area seen through attenuated retinal pigment epithelium.

Serum ornithine level was $241 \mathrm{mcmol} / \mathrm{l}$ (Gyrate atrophies tend to have values between 400 and $600 \mathrm{mcmol} / \mathrm{l}$ ).

Electrodiagnostic tests on her only son showed normal ERG responses to blue, red, white and flicker without evidence of a retinal lesion.

\section{Comment}

Since Kinross-Wright described a case of retinal pigmentary degeneration with the use of NP-207 in $1956^{4}$ many reports have surfaced almost invariably associating toxicity with high daily doses of thioridazine and only a few reports have been published relating pigmentary disturbances at lower doses. Recently a case was reported where a patient developed fine pigmentary stippling in both retinas with a total maximum daily dose of $400 \mathrm{mg}$ and a total dose of $752 \mathrm{~g}^{2}$. The case we present, 
which seemed to have had a progressive course after the drug was discontinued ${ }^{3,5}$ raises once more the point that there may be no safe dose for thioridazine and that the extent of damage may not correlate with the dosage and duration of treatment. It may also support the idea of toxicity due to enzyme inhibition $^{6}$ although saturation of melanin granules by other drugs with subsequent cumulative/synergistic toxicity by thioridazine absorbed by the uveal melanocytes ${ }^{7}$ still remains an interesting proposition.

M. Sousa Neves FCOphth DO

Kerry Jordan FRCS FCOphth

Henk Dragt MCOphth

West Suffolk Hospital

Bury St. Edmunds
References

${ }^{1}$ Morton Grant: Toxicology of the Eye. 2nd ed. Illinois. Charles Thomas, 1974: 1005-10.

${ }^{2}$ Lam RW and Remick RA: Pigmentary retinopathy associated with low dose thioridazine treatment (letter). Can Med Assoc J 1985, 132: 737.

${ }^{3}$ Davidorf FH: Thioridazine pigmentary retinopathy. Arch Ophthalmol 1973, 90: 251.

${ }^{4}$ Kinross-Wright V: Clinical trial of a new phenothiazine compound: NP-207. Psychiatr Res 1956, 4: 89-94.

${ }^{5}$ Meredith TA, Asberg TM, Willerson WD: Progressive chorioretinopathy after receiving thioridazine. Arch Ophthalmol 1978, 96: 1172-6.

${ }^{6}$ Weekley RD, Potts AM, Reboton J, May RH: Pigmentary retinopathy in patients receiving high doses of a new phenothiazine. Arch Ophthalmol 1960, 64: 65 .

${ }^{7}$ Potts AM: Uveal pigment and phenothiazine compounds. Trans Am Ophthalmol Soc 1962, 60: 517. 